\title{
Mobile Agent based Workflow Management System (MAWFMS) for Information Flow and Business Processes
}

\author{
RAHUL SINGH CHOWHAN*
}

Department of Computer Science, Agriculture University, Jodhpur, Rajasthan, India.

\begin{abstract}
Mobile agents are a very novice field with wider scope of research in the stream of a integrated coherent networks, parallel programming, automated learning, remote execution and artificial intelligence. A mobile agent is a representative that is often treated as moving object developed using any object oriented programming language. Using a WFMS is an emerging current trend in modeling, research methodologies and implementing the information/data flow and various related business processes within an organization. Work is often credited as a task, information, a document, data, announcements or a job that passes through different nodes within an organization. The integration of WFMS involves the automation of various processes such as tasks, documents, processed data, and information or rules to accomplish a task. This automation within the technology can be achieved by integration of agent based technology that makes use of Mobile Agent Paradigm. This paper briefs about various features, benefits and role mobile agents in Workflow management system. This also highlights how the implementation of MAWFMS can smoothen and simplifies the workflow managements in multifarious event based environments.
\end{abstract}

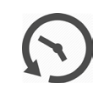

Article History

Received: 28 February 2018

Accepted: 23 March 2018

\section{Keywords}

Distributed computing, Process automation, Agent paradigm, Parallel programming.

\section{Introduction}

A mobile agent is an essential feature of programlogic and data that is capable of travelling between nodes in a network. Agent mobility scale states various properties for static and mobile agents. Mobility is one such orthogonal property which allows any agent to be movable within a fixed itinerary. If an agent is movable, i.e. it shows some hopping pattern, only then it will be denoted as mobile agent ${ }^{1}$. A static agent can just stay at one place and interacts with its underlying environment using conventional means of communication methods such as different configurations of Remote Procedure Calling (RPC), Remote Method Invocation (RMI), and other messaging mechanism while the mobile agent is not restricted to execute and work only on the context it is instantiated. It is also independent of travelling among the various inter-

CONTACT Rahul Singh Chowhan word2rahul@gmail.com $\mathbf{9}$ Department of Computer Science, Agriculture University, Jodhpur, Rajasthan, India.

(C) 2018 The Author(s). Published by Oriental Scientific Publishing Company

This is an $\bigcirc$ Open Access article licensed under a Creative Commons Attribution-NonCommercial-ShareAlike 4.0 International License (https://creativecommons.org/licenses/by-nc-sa/4.0/), which permits unrestricted NonCommercial use, distribution, and reproduction in any medium, provided the original work is properly cited.

To link to this article: http://dx.doi.org/10.13005/ojcst11.01.08 
connected nodes forming an itinerary ${ }^{2}$. After being created in one running context, it can take along its data state, execution state and business logic to next running context within the itinerary to resume/ restart the assigned job for purpose of monitoring resources or data collection. Most of the information based systems executes individual tasks whereas when it is integrated with mobile agent technology it allows coordinating, mutual execution, collaboration and monitoring of various tasks, host and resources within the interconnected network nodes. The WFM is rapidly advancing technology that invokes the need and primarily focuses on automation processes based on human and machine interactions. This system depicts a critical issue to obtain enterprise competitiveness among various firms. It also offers procedure based automation of any business process. This helps dealing of interrelated sequential processes and the invocation of proper human and IT resources associated with the various activity steps.

Prevalently, this system can be used within the organizational environment in member intensive and intricate processes like insurance, banking, legal and general administrations, etc, it is also applicable to various sections and sub-classes of manufacturing \& production based applications ${ }^{3}$. Mobile Agent based Workflow Management System (MAWFMS) allows/ needs inculcation of other technologies within in like agent technology, that facilitate flexibility, autonomy, and intelligent solutions for IF (information flow) and BPM (business process management).

\section{Features of Mobile Agents in Work Flow Management System Increased Efficiency}

Mobile agents serve effectively and efficiently being an unanimous working unit as single multifunctional integrated system. A client-side representative know-how moves on to the network admin or server-node. This is capable of making independent communication with a server program residing on admin node. After accomplishing the required goal it reverts back to the home node with a finished conclusion or an appropriate outcome. This local way of communicating with nodes in network drastically reduces the intermediate network traffic as the agents use local resources of migrated node.

\section{Greater reliability}

It boosts up the overall reliability off system because the connection between nodes is not required to stay online \& active all the time. Whereas with the previous architecture and models dedicated line was required to communicate which may have to deal with various faults due to congestion problems.

\section{Implementation level Uniformity}

The system of mobile agents can entertain development of a WFA (workflow administration) \& WFM (workflow monitoring) tools, organizing client applications and implementation of enactment services ${ }^{4}$. Agents can also be used as ubiquitous interface to invoke required applications and various workflow enactment services. There are various perspectives in organizing and implementing WMS which may introduce distinct tools, techniques \& procedures for implementation various modules within a system.

\section{Target Oriented}

Mobile agents are the work/task oriented entities that are passed on or moved to different nodes autonomously. Mobile agent server takes care of the current status i.e. execution state, data state and life cycle methods and itinerary planning of target oriented mobile agent. They are target oriented i.e. assigned with a particular task to accomplish, and they return to home agency when the given task is achieved. There exist various approaches that make use of distinct mechanisms to pass on the work-items (transactions, e-mails, message handles, etc.) and to keep track records of their current status (centralized control, repository status, database management etc.)

\section{Itinerary Planning}

The execution of work and its hopping nature can easily be handled and maintained by using mobile agents. It can easily be achieved by altering the mobile agent itineraries or introducing new mobile agents within the agent system. The structure of any organization or company is implicitly confined within the preplanned agent itineraries which can be manipulated on the fly giving instructions of agent. Whereas many other traditional approaches demands for bulky changes in central administration or one point access to achieve the same objectives ${ }^{5}$. 


\section{Agent Cloning}

The implementation of workflow system using mobile agents can make it easy to grow even being with existing complexities of its business processes running with underlying company. This type of cases just needs involvement and interaction of more agents. This could be made possible without any need to change the previously maintained system and without understanding the non-participatory integrated components and modules. The super agent simply replicates using cloning lifecycle method to distribute various tasks to sub-agents or cloned agents.

Table 1: Functionality and Benefits of MAWFMS

\begin{tabular}{lll}
\hline Functionality & IT Benefits & Business Benefits \\
\hline Self Service & Give Business user & Reduce Costs and Deliver \\
& Visibility Into Workload & unprecedented service \\
Batch Impact Manager & Proactively evaluate impact & Ensure on-time completion of \\
& of delays or failure & Batch Processes \\
Advance File Transfer & Integrate file transfer flow & Ensure Data Integrity and \\
& and batch scheduling & minimize risks \\
Forecast & Predict the expected flow & Minimize disruption to critical \\
& batch processes & services \\
Application Integration & Centralized Management & Increase availability and \\
& and Monitoring & Eliminate costs \\
\hline
\end{tabular}

Role of Mobile Agent in Workflow Management System

The movement of mobile agents in an itinerary is certainly non-identical than OS based process migration. The processes at operating system level are invoked on occurrence of any specific event like Interrupt, Clicks, etc. which results in transfer of control from current line of execution to the calling mechanism whereas the mobile agents are capable of initiating the process of migration on their own and continue their execution from previous states. Also there lies difference between the remotely executed program (such as Java Applets \& Servlets) and mobile agents because mobile agent can be an active/passive autonomous entity. The remotely executed program gets executed on the server and only carries results to the clients that invoke it. The mobile agent mechanism allows the migration of process state, which accompanies it to next hops. The workflow management using mobile agent to handle distributed data, heterogeneous resources, and proper distribution of workload and scalability factor can be covered under four categories ${ }^{6}$ :
Workflow Agent Development Environment (WADE)

This entertains the production engine of mobile agents and required set of options to define various processes related to workflow management system. The definition tools of development processes includes process analysis, synthesis, modeling, process definition, other process related information. It also differentiates between process behaviors \& objects and outcasts a meta-data model that defines behaviors and objects ${ }^{7}$. The ADM (agent developing module) is responsible for design and programming of mobile agent as per the directives from definition tools defined within the development processes, and puts the subsequent agent to WME.

\section{Workflow Management Environment (WME)}

This environment consists of a module of workflow management module (WFMM). This also has persistent home-base for agents. The main task of this system is to allow and receive workflow agents from the development process environment. Then it saves the states of various agents in the agent 
home-base. It responds to various requests of customers side by side staying connected to the Internet connection. It also fetches the successive WFA (workflow agents) lying within the home-base and sends them to MA execution environment. It also displays the agent workflow status on an interface that is obtained from the called agents to monitor the workflow.

\section{Mobile Agent Runtime Environment (MARE)}

This is the runtime module that supports the necessary mechanism to allow various facilities of the mobility, on-call or self-execution, management of mobile agent associated methods. This supports initialization of WFMA and can also request \& respond it using ATI (agent transport interface) using agent transport protocol (ATP). The runtime environment also supports the mutual interaction of various agents and merging with communities sharing similar interests. They may also involve external workflow participants like humans or some interactive tools of various applications ${ }^{8}$. This mobile agent runtime environment allows the stand-alone platform by using virtual machine for mobile agents in a heterogeneous network.

\section{Mobile Agent Server Service \& Execution Environment (MASSEE)}

Bascially the context which allows and maintains activities related to mobile agent like Owner name, travel history, goal to achieve etc. It also takes care for serialization and desearilization of mobile agents as required. It wakes up the mobile agent for execution to be resumed or restarted as per the business logic available within the agent. It also maintains a repository for status management of various life cycle states of mobile agent like creation, dispatch, retract etc as shown in Fig 1. This is the compulsory component of mobile agent system to allow any mobile agent for navigation to remote hosts ${ }^{9}$. It also keeps the information of clients like thin client or thick client as per the requirements mentioned for executinon of mobile agents in any context.

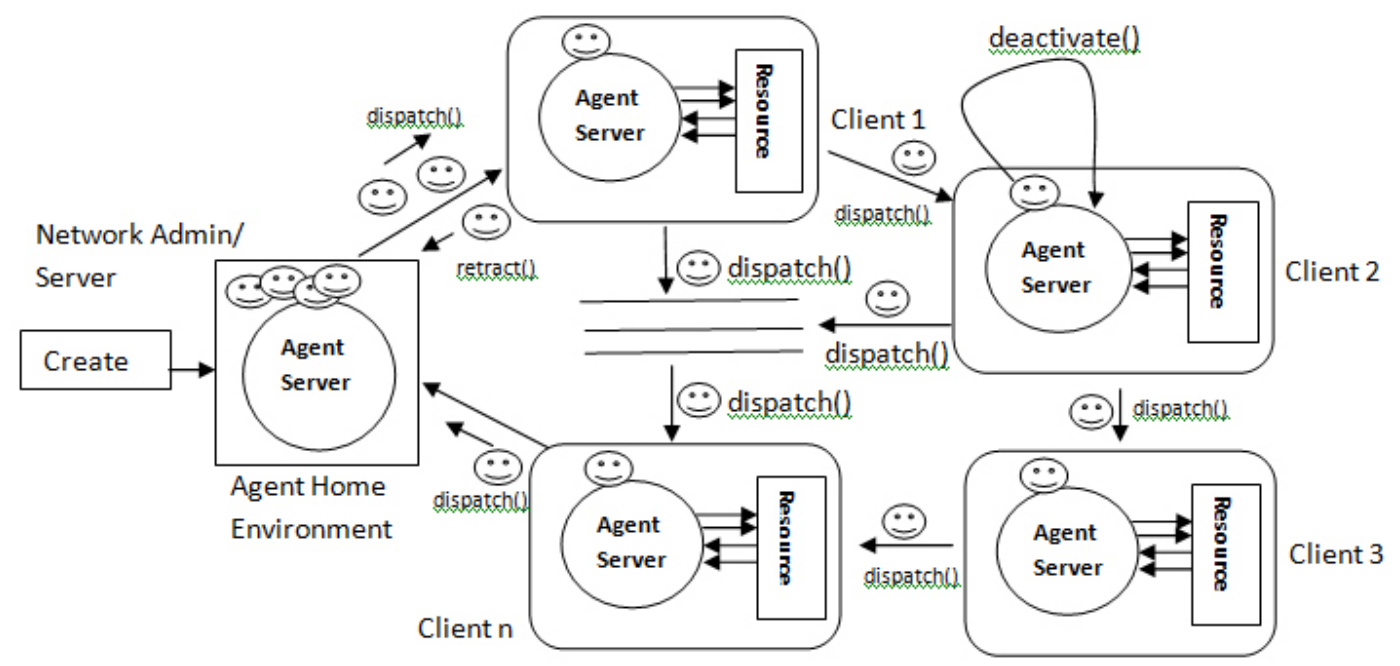

Fig. 1: Working model of Mobile Agent

\section{Current status and Drawbacks}

The foundation of Workflow's lies in handling various business processes that are live and continuing in the various areas within the company to supply and fitting the incoming requests. The WFMS deals with following defined entities:

\section{Business Oriented Process}

These are the bundle of co-related jobs or actions collaborating to work for unique business objective. The contextual factors of an organizational framework allow running this process orientation. The objective of this orientation basically supports in defining 
various functional \& contextual roles. It also tells how the identities within the set are correlated and affecting one another.

\section{Organizational Workflow}

This is an automated mechanism for an objective based business process. Throughout the mechanism of workflow various processes, information jobs or works etc needs to be conveyed on from one participating entity to another within the predefined sequence to act as per the predefined protocols and a sequence of action history.

\section{Workflow Management System}

This is the module that describes, determines and supervises the workflow performance using monitoring software. The software is basically operating on one or many workflow engines simultaneously. It has capability of interpreting the process definition and communicates with participating identities within the workflow. It can also invoke appropriate application tools as and when required ${ }^{10}$.

The above WFMM (workflow management model) can be applied for configuring analytical \& soundly efficient enterprises. Nevertheless, this framework has loopholes in handling \& managing the business processes:

\section{Lack of Support to Sustain Loose-Cooperating Framework}

The business process participating in e-commerce and $\mathrm{m}$-commerce involves communication of many worldwide organizations. Many times while commencing online communications, organizations may not be interested in binding up their interest and support for participants as they do with partners working in the same company. The benefit that any loosely cooperating framework avails is that its processes can be adjusted as per the objectives and purposes for business profit of entities. The above WFM (workflow management) model does not configure such loose-cooperating framework.

\section{Lack of the Unanimous Communication Platform}

Internet is the web of multifarious commercial companies which make use of dissimilar communication platform, agent server architecture, workflow management software, communicative model and interaction software kit. This heterogeneous nature of internet makes it much difficult to deploy workflow applications.

\section{Lack of the Support to Local Autonomy}

The workflow processes are required to be defined and deployed in advance for the workflow management model discussed above. This makes the loss of local autonomy for the participants communicating within this system ${ }^{11}$. This might be acceptable for the participants within the single enterprise but it is not suitable for loose-cooperating partners communicating via e-commerce or $\mathrm{m}$-commerce. This is because inter enterprise communication demands for some local autonomy mechanism to look on incoming exceptions for purpose of profit.

\section{Major Issue of Security}

Security is the major challenging issues of all which needs immediate attention while implementing the mobile agent in workflow management. As mobile agents is an executable code which travels across the network making it easy security breaches. This requires self-protecting mechanism to be build within the mobile agent against the hostile hosts that would try to manipulate, modify and obtain information carried along with the mobile agents. The intergalactic roaming property of a mobile agent and ability of on the fly execution on a foreign server system makes it vulnerable to malicious attacks from other agents and servers ${ }^{12}$. The few broad areas dealing with security of mobile agent are stated as under:

\section{Protection of Non-Malicious Network Hosts from Malicious Mobile Agents}

It may occur within the unrestricted environment of interconnected nodes, such as Internet, that the host might introduce the mobile agent with mala-fide intention. This requires the proper security measures at the host machine allowing various mobile agents from/within the network. The mobile agent server called as agency, which entertains the mobile agent on the target host should check the nature of tasks assigned to the mobile agent and takes proper monitoring of execution of agent within the restricted environment. 
Protection of Non-Malicious Mobile Agents from Malicious Network Hosts

In agent based technology, the malicious host is stated as a node that not only allows execution of a mobile agent but also tries to attack the agent. Whenever any mobile agent gets executed on a host it makes use of underlying available resources with the host ${ }^{13}$. This way node can monitor actions of agent like instructions it execute, memory consumed, type of task it carries etc. and may attack. Few frequent attacks that a malicious host can carry out on mobile agent can be:

- $\quad$ The malicious host masquerading as nonmalicious host.

- DoS attack on the agent by the host.

- Eavesdropping and tracking activities of agent.

- $\quad$ Forcing the changes in implementation of mobile agent by the malicious network host.

The safety measures that can be taken against the above attacks based on two main information criteria of mobile agents:

\section{Identification and Detection}

- Detecting the modification of business logic, execution state, data state etc.

- Execution tracing and also tracking the itinerary of agent

- $\quad$ Acquiring the information of current execution environment

- $\quad$ Partial Result Encapsulation using various cryptographic techniques like digital signature, hash functions etc.

\section{Prevention}

- $\quad$ Reverting the unauthorized access to code of mobile agent

- Use of encrypted function i.e. embodying of original function within the enciphered function

- $\quad$ Pre-execution checking of mobile agent on its arrival

- $\quad$ Skimming through the previous itinerary traces of an agent

- $\quad$ Setting up the authenticated channel for agent navigation
- $\quad$ Installing fault tolerant mechanism with agent server

- Working within the closed loop of trusted nodes.

\section{Portability and Standardization}

The mobile agents are portable entities and that could do both weak as well as strong migration depending on need of tasks and goal assigned to restart or resume them. So the migration of mobile agent makes sure that it should be engaged on the completely hostile environment i.e. the mobile agent developed in one object oriented language could be entertained by platform of another object oriented language. At same time, it should follow a standard mechanism to ensure high security whether provided by programming language or by the platform of execution.

\section{Performance and Scalability}

The introduction of multiple mobile agents on single platform or agent sever, whether generated for different purposes or cloned to achieve one single goal, could decrease the performance and increases the security risks ${ }^{14}$. It supports the scalability factor and works to congregate the scattered information in a relevant manner which would further extend the increase of security breaches as mobile agent migrates from one node to other within the itinerary.

\section{Benefits of Using Mobile Agent for Workflow Management}

The mobile agent workflow management model that makes use of various kinds of mobile agents for flow and business processes is well suited for automated maintenance of workflow within the organization. The few merits are as under:

\section{Heterogeneity in Mobile Agent Distribution Platform}

The weak or strong migration and the implementation of mobile agents are dealt by the execution context of Mobile Agent. The workflow management agents are not dependent on the current system platform where the agent has been created, this feature allows it to make virtually active ${ }^{15}$. Each participatory node within WFM requires the running Agent Runtime 
Environment (ARE), also called as Agent Server. The Workflow Management Engine is just required at the server node which is responsible to handle the various task assignment and execution related activities of Mobile Agent.

\section{Supports the Migration and Execution Level Autonomy}

Autonomy is one of the essential characteristic of a mobile agent which allows any mobile agent to migrate and execute on remote interconnected nodes within the network. The mobile agents working in this system has the capability to choose and operate on the next hop as per its own present status and the state of its current execution environment. This property makes the mobile agent to act and operate with the loosely coupled architectures by altering either local data or work plan.

\section{Reinforces Concurrent Computing}

The static or mobile agent is capable of spawning several child agents concurrently, to minimize the overall processing carried out on single resourceful node. The concurrent formation and computation of mobile agents is highly dependent on its current context, execution state and assigned tasks ${ }^{16}$. These child-agents may spread across the machines looking for more available resources to reduce the load on one machine. They also have capability of merging again with the master static or mobile agent on accomplishing the tasks assigned by the master agent.

\section{Loosely Coupled and Cooperating Architecture} The internet activities require a high loosely-coupled architecture for less-time-open connection based communications. The traditional workflow model, mostly matches the roles of appropriate participating nodes with the workflow management application. Whereas the final deployment of the application is implemented in the main process of execution, also called as process definition. These parameters are predefined before the execution so if any participant alters any of the predefined parameters then whole action is required to be carried out again. The introduction of mobile agent based autonomous working in the current workflow model allows matching process to be lazy loaded to WFR. This makes it more opportune to calibrate WFM procedure to alter loosely cooperating framework ${ }^{17}$.

\section{Workflow Recovery and Fault Tolerance Mechanism}

With mobile agent's being interference in the traditional WFMS benefits like persistence storage, encapsulation of execution state etc. has become possible. The serialization of mobile agents on the persistence storage of the current context enables mobile agents working in this system to consistently be available on accessible persistent storage. It makes backup mechanism more comprehensible $\&$ autonomous. Mobile agent workflow system also boosts fault tolerance developing sustainability of the overall with retracting, resuming and restarting nature.

\section{Applications Areas Of Mobile Agent Based On Workflow Management System}

Mobile agent serves various applications in numerous dimensions of computer science and information technology. The mobility factor of agents makes it suitable for various areas and domains even for fields where conventional techniques are used. The few of applications are as follows:

\section{E-Commerce or M-commerce}

The agent based architecture is well suited for continuously changing and activity oriented platforms like e-commerce. At many times a commercial transaction may require real-time access to remote resources like transaction history etc as well as agent-to-agent negotiation. In multi-agent system, various agents participate and compete for different goals. They may take use of different strategies or devise their own learning mechanism to meet these goals ${ }^{18}$. A goal oriented agent encapsulates the intention of its owner for which it act and negotiate on their behalf. MAWFMS is a very appealing solution to this kind of problem where generalized flow of data can be synchronized based on its type and requirement.

\section{Self Assisting Personal Assistance}

The ability of mobile agent to get executed remotely makes it favorable to be used as an assistant. The personal assistant i.e. the mobile agent is able to activate/deactivate jobs on remote machine in network on behalf of owner. This kind of mobile agent can operate independently acting as an assistant on the remote host maintaining its life cycle methods. It can communicate locally with its limited 
network connectivity and without any necessity of the owner being online and connected all the time ${ }^{19}$. The network admin can assign a task of scheduling a meeting to mobile agent, which may interact with other users or representative agents of users intended to attend the meeting. This assistant agent can also negotiate on various scheduling details like venue, timing, topic, terms etc. and finalize it.

\section{Agent based Distributed Information Retrieval}

The one of the famous application of mobile agent includes Information retrieval. With the increase in data, information and communication channels, processing and retrieval of information is becoming more tedious. Mobile agent provides a convincing idea by dispatching agents to remote information sources. Agents generate search indexes on remote sources visited by them, which are reverted to home agent server. This drastically reduces the moving of bulk of search results to search engines just for the purpose of indexing and ranking. Mobile agents are capable of advanced searches using deep learning methods without constraining the home server when it is operational. This allows the home server of mobile agents to be free to handle other server level activities.

\section{Workflow Applications}

This has become a mandatory property for a general workflow application to assist course of data knowledge among co-workers within one enterprise. The mobile agents are beneficial in this case, as along with mobility they also serve with a degree of autonomy to the workflow item. Mobile agent based independent workflow items are the individual entities that embodies the information and behavior of task. This encapsulated information guides them to move within the constrained environment of an enterprise independent of any particular action, input and application.

\section{Concurrent Computing and Parallel Processing}

The mobile agent system is capable of creating the cascade of agent clones in the network for purpose of distribution of task. The main agent or parent agent can potentially administer and monitor parallel processing tasks assigned to various clones or child agents ${ }^{20}$. Mobile agents can do computation \& processing intensive tasks with this ability of performing distribution of tasks among multiple processors. This makes it a promising dynamic infrastructure for the hosts with less computing and processing resources.

\section{Virtual Platform Support}

Mobile agents are efficient in working and handling the virtual environment related activities. They can migrate over the machine servicing different requests $^{21,22}$. They can be used for load monitoring, load balancing and load sharing purposes. They can also be implemented with the various load scheduling algorithms to ensure the efficient handling of incoming and outgoing request along with migration of jobs/tasks so that no machine sits idle while other is pipelined with numerous waiting jobs/tasks in the virtual environment of connected machines.

\section{Conclusion}

Mobile agent system is equally capable to accomplish anything that is possible with conventional methods. Mobile agents help reducing the overall execution time and resource consumption on single machine making tasks easier via concurrent computing. Mobile agents are efficient in dealing with real-time systems as they can interact and decide while executing in current context in favor of the assigned goals. Mobile agents can migrate to the node that it needs to operate on for time-crucial tasks, which removes the delays caused by sending messages between a client and a server.

In this paper, it is been presented the main motivation for using mobile agents in workflow management. Mobile agents being an independent and autonomous entity can navigate to multiple locations using IP-addresses and can execute on host machines. As this system requires some dynamic entity which can manipulate and take decisions by itself. It possesses vast future scope in field of workflow management where multiple events occur simultaneously so spontaneous decision making is required. Mobile agent technology is promising and emerging paradigm that brings some important advantages over traditional client/server solutions in workflow management system.

\section{Acknowledgemnt}

First, my sincere thanks to Late Prof. (Dr.) Rajesh Purohit, without whom my interest for mobile agent 
technology would have not flourished. Further, it is grateful for me to extend my regards to all academic friends and lecturers who have helped me in completing this refined work. They are (alphabetical order) Ashish Sharma, Poonam Purohit, Purva Dayya, Shilpa Kumari and Shivam Lohiya.

\section{References}

1. Chowhan, Rahul Singh and Purohit, Rajesh. "Study of Mobile Agent Server Architectures for Homogeneous and Heterogeneous Distributed Systems." International Journal of Computer Applications, 2016.

2. Yan, Yuhong., Zakaria, Maamar., and Shen, Weiming. "Integration of workflow and agent technology for business process management." In Computer Supported Cooperative Work in Design, The Sixth International Conference on, 2001, pp. 420426. IEEE, 2001

3. Silva., Moura, Luis et al., "The performance of mobile agent platforms." Agent systems and applications, 1999 and third international symposium on mobile agents. Proceedings. First international symposium on. IEEE, 1999.

4. Chowhan, Rahul Singh and Purohit, Rajesh. "Monitoring of Distributed Resources Based on Client-Server (single-hop) Mobile Agents in Homogeneous Network of Interconnected Nodes." In International Conference on Information and Communication Technology for Intelligent Systems, pp. 25-32. DOI: 10.1007/978-3-319-63645-0_3 Springer, Cham, 2017.

5. Gang, Wu., Quanyuan, Wu and Huaimin, Wang. "A novel workflow management model based on mobile agents for internet electronic commerce." In Technology of Object-Oriented Languages and Systems, 2000. TOOLSAsia 2000. Proceedings. $36^{\text {th }}$ International Conference on, pp. 182-187. IEEE, 2000.

6. Chowhan, Rahul Singh., Karel, Archna and Kumari, Shilpa. "Intelligent Mobile Agent Based Context Aware E-Health Monitoring and Information Retrieval." Int. J. Pure App. Biosci 5, no. 6 (2017): 1037-1041

7. Huang, Ching-Jen., Trappey, Amy JC and Yao, Yin-Ho. "Developing an agent-based workflow management system for collaborative product design." Industrial Management \& Data Systems 106, no. 5 (2006): 680-699.

8. Quelhas, de Oliveira., Curado, Nuno. "Optimization of the workflow of external tools for customer support services.” (2017).

9. Chowhan, Rahul Singh., Mishra, Amit and Mathur, Ajay. "Aglet and kerrighed as a tool for load balancing and scheduling in distributed environment." In Recent Advances and Innovations in Engineering (ICRAIE), 2016 International Conference on, pp. 1-6. IEEE, 2016

10. Bellavista, Paolo., Corradi, Antonio and Stefanelli, Cesare. "How to monitor and control resource usage in mobile agent systems." In Distributed Objects and Applications, 2001. DOA'01. Proceedings. $3^{\text {rd }}$ International Symposium on, pp. 65-75. DOI: 10.1109/ DOA.2001.954072, IEEE, 2001.

11. Srinivasan T., kumar, Vivek Vijay and R. Chandrasekar. "A self-organized agentbased architecture for power-aware intrusion detection in wireless ad-hoc networks." In 2006 International Conference on Computing \& Informatics, pp. 1-6. DOI: 10.1109/ ICOCI.2006.5276609 IEEE, 2006.

12. Kaur, Maneet and Sharma, Sandeep. "A dynamic clone approach for mobile agent to survive server failure." In Reliability, Infocom Technologies and Optimization (ICRITO) (Trends and Future Directions), $20154^{\text {th }}$ International Conference on, pp. 1-5. DOI: 10.1109/ICRITO.2015.7359237, IEEE, 2015.

13. Hans, Rahul and Kaur, Ramandeep. "Novel dynamic shadow approach for fault tolerance in mobile agent systems." In Signal Processing and Communication Systems (ICSPCS), $20126^{\text {th }}$ International Conference on, pp. 1-6. DOI: 10.1109/ICSPCS.2012.6507945, IEEE, 2012.

14. Lingaraj. K., V. Biradar, Rajashree and 
Patil, V. C. "A survey on mobile-agent middleware design principals and itinerary planning algorithms." In 2015 International Conference on Applied and Theoretical Computing and Communication Technology (iCATCCT), pp. 749-753. DOI: 10.1109/ ICATCCT.2015.7456983, IEEE, 2015.

15. Hans, Rahul and Kaur, Ramandeep. "Location tracking in mobile agents system using forward and backward pointers." In Engineering and Computational Sciences (RAECS), 2014 Recent Advances in, pp. 1-5. DOI: 10.1109/ RAECS.2014.6799508, IEEE, 2014.

16. Channappagoudar, Mallikarjun B. and Venkataram, Pallapa. "Mobile agent based node monitoring protocol for MANETs." In Communications (NCC), 2013 National Conference, pp. 1-5. DOI: 10.1109/ NCC.2013.6488044, IEEE, 2013.

17. Trivedi, Kartik and Srivastava, Ashish Kumar. "An energy efficient framework for detection and monitoring of forest fire using mobile agent in wireless sensor networks." In Computational Intelligence and Computing Research (ICCIC), 2014 IEEE International Conference, pp. 1-4. DOI: 10.1109/ICCIC.2014.7238433, IEEE, 2014.

18. Eldegwi, H. M. ., Badawy, M. B. ., and Kelash, Hamdy M. "Building a Secure Decentralized
Energy System with Remote Monitoring Using Mobile Agents." In 2015 Fifth International Conference on e-Learning (econf), pp. 263268. DOI: 10.1109/ECONF.2015.80, IEEE, 2015.

19. Jo, Mi Young and Kim, Keecheon. "A research on the regional routing scheme based mobile agent for SDN." In 2016 International Conference on Information Networking (ICOIN), pp. 211-213. DOI: 10.1109/ICOIN.2016.7427116, IEEE, 2016.

20. Kumar, Rajesh., Niranjanr, S and Singh, Yashpal "A Review on Mobile Agent Technology and Its Perspectives." Journal of Computer Sciences and Applications, vol. 3, no. 6: 166-171. DOI: 10.12691/jcsa-3-6-11, (2015).

21. Fragkakis, Michail and Alexandris, Nikolaos. "Comparing the trust and security models of mobile agents." Information Assurance and Security, 2007. IAS 2007. Third International Symposium on. IEEE, 2007.

22. Trillo, Raquel., Ilarri, Sergio and Mena, Eduardo. "Comparison and performance evaluation of mobile agent platforms." Autonomic and Autonomous Systems, 2007. ICAS07. Third International Conference on. IEEE, 2007. 